\title{
On the group of circular units of any compositum of quadratic fields
}

\author{
by \\ ZDENĚK POLICKÝ (Brno)
}

Introduction. The aim of this paper is to describe the group of circular units $C$ of a compositum $k$ of quadratic fields in the last case that has not been covered yet, namely when the ramification index $e$ of 2 equals 4 . It is easy to see that $e$ always divides 4 . If $e=1$ or $e=2$ we already know a basis of $C$ and an explicit formula for the index of $C$ in the full group of units $E$ (see [2, Theorem 1] and [4, Proposition 1.4]). The main ingredient of these results was the observation that the action of the augmentation ideal of $\mathbb{Z}[G]$, where $G=\operatorname{Gal}(k / \mathbb{Q})$, on the quotient $C / W$, where $W$ is the group of all roots of unity in $k$, gives squares in $C / W$. In other words, for any $\varepsilon \in C$ and any $\sigma \in G$ there is $\rho \in W$ and $\eta \in C$ such that $\varepsilon^{1-\sigma}=\rho \eta^{2}$. Unfortunately, this key property of the group of circular units of a compositum of quadratic field is not satisfied in the case $e=4$ (see Example 8 below for $k=\mathbb{Q}(\sqrt{-1}, \sqrt{2}, \sqrt{-3}))$. Therefore if $e=4$ we cannot use the same approach for $k$. Nevertheless, using the three maximal subfields of $k$ whose ramification index at 2 is 2 , we are able to describe an explicit maximal independent system of units in $C$. Let $\tilde{C}$ be the group generated by $W$ and by this system. Then we can compute the index $[E: \tilde{C}]$ and give a reasonable upper bound for the index $[C: \tilde{C}]$ (see Theorem 7 and Proposition 5).

1. Definitions and basic results. Let $k$ be a compositum of quadratic fields and let $K$ be the genus field of $k$ in the narrow sense. We assume that both -1 and 2 are squares in $K$. We put

$$
J=\{-1,-2,2\} \cup\{p \in \mathbb{Z} ; p \equiv 1(\bmod 4),|p| \text { is a prime ramifying in } k\} .
$$

2000 Mathematics Subject Classification: Primary 11R29; Secondary 11R27.

Key words and phrases: compositum of quadratic fields, circular units, the index of circular units, class number. 
For any $p \in J$, let

$$
n_{\{p\}}= \begin{cases}|p| & \text { if } p \notin\{-1,-2,2\} \\ 4 & \text { if } p=-1, \\ 8 & \text { if } p= \pm 2 .\end{cases}
$$

For any $S \subseteq J$ let $n_{S}$ be the smallest common multiple of $n_{\{p\}}$ for all $p \in S$ (by convention $n_{\emptyset}=1$ ), and

$$
\zeta_{S}=e^{2 \pi i / n_{S}}, \quad \mathbb{Q}^{S}=\mathbb{Q}\left(\zeta_{S}\right), \quad K_{S}=\mathbb{Q}(\sqrt{p} ; p \in S), \quad k_{S}=k \cap K_{S} .
$$

It is easy to see that $K_{J}=K, k_{J}=k$, and $n_{J}$ is the conductor of $k$.

We call a subset $S \subseteq J$ admissible if $S$ contains at most one of the numbers $-1,2$, and -2 . For any admissible set $S \subseteq J$ we define

$$
\varepsilon_{S}= \begin{cases}1 & \text { if } S=\emptyset, \\ i & \text { if } S=\{-1\}, \\ \frac{1}{\sqrt{p}} \mathrm{~N}_{\mathbb{Q}^{S} / K_{S}}\left(1-\zeta_{S}\right) & \text { if } S=\{p\}, p \neq-1, \\ \mathrm{~N}_{\mathbb{Q}^{S} / K_{S}}\left(1-\zeta_{S}\right) & \text { if } \#>>1,\end{cases}
$$

and $\eta_{S}=\mathrm{N}_{K_{S} / k_{S}}\left(\varepsilon_{S}\right)$.

Let $\chi_{2}$ and $\chi_{-2}$ be the unique even and odd Dirichlet character of conductor 8 , respectively. For each $p \in J-\{2,-2\}$ let $\chi_{p}$ be the unique Dirichlet character of conductor $n_{\{p\}}$, so $\chi_{p}$ is odd if and only if $p<0$.

Let $X$ be the group of all even Dirichlet characters corresponding to $k$. Each $\chi \in X$ can be written in the form $\chi=\prod_{p \in S_{\chi}} \chi_{p}$ for a unique admissible set $S_{\chi} \subseteq J$. Then the conductor of $\chi$ is equal to $n_{S_{\chi}}$.

It is easy to see that, for any admissible set $S \subseteq J$, a character $\chi \in X$ belongs to the set of Dirichlet characters corresponding to the field $k_{S}$ if and only if $S_{\chi} \subseteq S$.

Let $C$ be the group of circular units of $k$ defined in [3]. This means that $C$ is generated by $W$ and by all conjugates of $\eta_{S}$, where $S \subseteq J$ (see the proof of Proposition 4 below). This group contains the Sinnott group of circular units $C^{\prime}$ of $k$ but it can be slightly bigger. Lemma 3 in [2] implies that the Sinnott group is generated by

$$
W \cup\left\{\eta_{S} ; S \subseteq J, \# S>1\right\} \cup\left\{\eta_{p}^{2} ; p \in J, p>0, \sqrt{p} \in k\right\}
$$

and consequently $\left[C: C^{\prime}\right]=2^{a}$, where

$$
0 \leq a \leq \#\{p \in J ; p>0, \sqrt{p} \in k\} .
$$

Similarly, for any $S \subseteq J$ let $C_{S}$ be the group of circular units of $k_{S}$ defined in [3]. If $S$ is admissible then the ramification index of 2 in $k_{S}$ is not equal to 4 and so we know the following basis of $C_{S}$ :

Lemma 1. If $S \subseteq J$ is admissible then a basis of $C_{S}$ is formed by the set of all $\eta_{S_{\chi}}$ where $\chi \in X$ is non-trivial and satisfies $S_{\chi} \subseteq S$. 
Proof. If $-1 \notin S$ see [2, Lemma 5], otherwise see [4, Proposition 1.4].

Let $W$ be the group of all roots of unity in $k$. Let $\tilde{C}$ be the subgroup of the multiplicative group $k^{\times}$generated by $W$ and by all conjugates of $\eta_{S}$ for all admissible sets $S \subseteq J$. Let $G=\operatorname{Gal}(k / \mathbb{Q})$ be the Galois group of $k$.

Lemma 2. For any $\varepsilon \in \tilde{C}$ and any $\sigma \in G$ there are $\rho \in W$ and $\eta \in \tilde{C}$ such that $\varepsilon^{1-\sigma}=\rho \eta^{2}$.

Proof. Consider a conjugate of $\eta_{S}$ for an admissible set $S \subseteq J$. If $-1 \notin S$ use [2, Lemma 2], otherwise use [4, Lemma 1.2].

Lemma 3. The set $W \cup\left\{\eta_{S_{\chi}} ; \chi \in X, \chi \neq 1\right\}$ generates the group $\tilde{C}$.

Proof. Lemma 2 gives that $\tilde{C}$ is as a group generated by $W$ and by $\eta_{S}$ for all admissible sets $S \subseteq J$. For any admissible set $S \subseteq J$ we can show that if $S \neq S_{\chi}$ for all $\chi \in X$ then $\eta_{S}$ can be written as a multiplicative $\mathbb{Z}$-linear combination of $\eta_{L}$ for $L \subsetneq S$ (modulo roots of unity). If $-1 \notin S$ use [2, Lemma 5], otherwise use [4, p. 1077].

\section{The index of $\tilde{C}$ in $C$}

Proposition 4. The group $C$ of circular units of $k$ is generated by $\tilde{C}$ and by all conjugates of $\mathrm{N}_{\mathbb{Q}^{S} / k_{S}}\left(1-\zeta_{S}\right)$, where $S \subseteq J$ is not admissible, $S \neq\{-1,2,-2\}$, and the ramification index of $k_{S}$ at 2 is 4 .

Proof. Let $E$ be the full group of units of $k$. By definition (see [3]), $C$ is the intersection of $E$ and a group $D$, where $D$ is generated by -1 , by $\sqrt{p}$ for all $p \in J$ such that $p>0$ and $\sqrt{p} \in k$, and by all conjugates of $\mathrm{N}_{\mathbb{Q}^{S} / k_{S}}\left(1-\zeta_{S}\right)$ for all non-empty $S \subseteq J$.

For a non-empty $S \subseteq J$, it is well-known that $\mathrm{N}_{\mathbb{Q}^{S} / k_{S}}\left(1-\zeta_{S}\right)$ is a unit if and only if $n_{S}$ is not a prime power. Moreover, if $p \in J$ and $p<0$ then all units of $k_{\{p\}}$ are roots of unity. Therefore $\tilde{C}$ is the intersection of $E$ and a group $\tilde{D}$, where $\tilde{D}$ is generated by -1 , by $\sqrt{p}$ for all $p \in J$ such that $p>0$ and $\sqrt{p} \in k$, and by all conjugates of $\mathrm{N}_{\mathbb{Q}^{S} / k_{S}}\left(1-\zeta_{S}\right)$ for all admissible non-empty $S \subseteq J$.

If $S$ is not admissible and the ramification index of $k_{S}$ at 2 is not 4 then $k_{S}=k_{S^{\prime}}$ for a suitable admissible $S^{\prime} \subseteq S$. Hence $D$ is generated by $\tilde{D}$ and by $\mathrm{N}_{\mathbb{Q}^{S} / k_{S}}\left(1-\zeta_{S}\right)$ for all non-admissible $S \subseteq J$ such that the ramification index of $k_{S}$ at 2 is 4 . This norm is a unit unless $S=\{-1,2,-2\}$ and $\sqrt{-1}, \sqrt{2} \in k$, in which case $k_{S}=\mathbb{Q}(\sqrt{-1}, \sqrt{2})$ is the eighth cyclotomic field. But the group of all units of the latter is generated by $\zeta_{8}$ and by

$$
\eta=\zeta_{8}^{-1} \cdot \frac{1-\zeta_{8}^{3}}{1-\zeta_{8}}=1+\zeta_{8}+\zeta_{8}^{-1}=1+\sqrt{2}
$$


We have

$$
\eta_{\{2\}}=\frac{1}{\sqrt{2}} \mathrm{~N}_{\mathbb{Q}\left(\zeta_{8}\right) / \mathbb{Q}(\sqrt{2})}\left(1-\zeta_{8}\right)=\sqrt{2}-1=\eta^{-1},
$$

and the proposition follows.

Proposition 5. The group $\tilde{C}$ is of finite index in $C$ and $[C: \tilde{C}] \leq 2^{n}$, where $n$ is the number of all $S \subseteq J$ such that $\{-1,2,-2\} \subsetneq S$ and the ramification index of $k_{S}$ at 2 is 4 . Moreover, the Galois action of $G$ on $C / \tilde{C}$ is trivial.

Proof. Let $T=J-\{-1,2,-2\}$. For any $x \in\{-1,2,-2\}$ let $\rho_{x}$ be the generator of $\operatorname{Gal}\left(K / K_{T \cup\{x\}}\right)$. For any $L \subseteq T$ we put $S=L \cup\{-1,2,-2\}$ and $\varepsilon=\mathrm{N}_{\mathbb{Q}^{S} / k_{S}}\left(1-\zeta_{S}\right)$. Then

$$
\varepsilon^{2}=\varepsilon^{1+\rho_{-1}} \cdot \varepsilon^{1+\rho_{-2}} \cdot\left(\varepsilon^{1+\rho_{2}}\right)^{-\rho_{-1}} .
$$

For any $x \in\{-1,2,-2\}$ we have

$$
\varepsilon^{1+\rho_{x}}=\mathrm{N}_{\mathbb{Q}^{S} / k_{T \cup\{x\}}}\left(1-\zeta_{S}\right)=\eta_{T \cup\{x\}}
$$

because $\mathrm{N}_{\mathbb{Q}^{S} / \mathbb{Q}^{T}\{\{x\}}\left(1-\zeta_{S}\right)=1-\zeta_{T \cup\{x\}}$. We have obtained $\varepsilon^{2} \in \tilde{C}$ and for any $\sigma \in G$ Lemma 2 gives $\varepsilon^{2(1-\sigma)} \in W \cdot \tilde{C}^{2}$, which implies $\varepsilon^{1-\sigma} \in \tilde{C}$. The proposition follows by means of Proposition 4.

\section{A basis of $\tilde{C}$ and the index of $\tilde{C}$ in $E$}

TheOrem 6. The set $\left\{\eta_{S_{\chi}} ; \chi \in X, \chi \neq 1\right\}$ is a $\mathbb{Z}$-basis of $\tilde{C}$, i.e. elements of this set are multiplicatively independent and together with $W$ generate $\tilde{C}$.

Proof. Proposition 5 gives that $\tilde{C}$ and $C$ have the same $\mathbb{Z}$-rank. As the index $[E: C]$ is finite, $\tilde{C}$ and $E$ have the same $\mathbb{Z}$-rank, and the $\mathbb{Z}$-rank of $E$ is equal to the number of elements of the given set. The theorem follows from Lemma 3.

Having a $\mathbb{Z}$-basis allows us to compute the index:

TheOREM 7. We have

$$
[E: \tilde{C}]=\left(\prod_{\chi \in X, \chi \neq 1} \frac{2 \cdot\left[k: k_{S_{\chi}}\right]}{\left[k: k^{+}\right]}\right) \cdot|X|^{-|X| / 2} \cdot Q h^{+},
$$

where $k^{+}$is the maximal real subfield of $k,|X|$ means the number of characters in $X, Q=\left[E: W \cdot\left(E \cap k^{+}\right)\right]$is the Hasse unit index of $k$, and $h^{+}$ is the class number of $k^{+}$.

Proof. This can be proved in the same way as Theorem 1 in [2]. 
The following example shows that the estimate of the index $[C: \tilde{C}]$ can be precise. It seems to be an interesting question whether this holds true in general.

EXAmPLE 8 . Let $k=\mathbb{Q}(\sqrt{-1}, \sqrt{2}, \sqrt{-3})$. Then $k$ is the 24 th cyclotomic field. Sinnott's formula for the index of the group of circular units of a cyclotomic field (see [5, Theorem]) shows that the Sinnott's group of circular units of $k$ equals $E$ and so we also have $C=E$. Then [1, Theorem 6.1] gives the following $\mathbb{Z}$-basis of $C: \alpha=1-\zeta, \beta=1-\zeta^{19}, \gamma=\left(1-\zeta^{9}\right) /\left(1-\zeta^{3}\right)$. As $\beta$ is a conjugate of $\alpha$, we see that we obtain $\alpha \cdot \beta^{-1}$ by an action of the augmentation ideal on $\alpha$. As both $\alpha$ and $\beta$ belong to a basis we see that $\alpha \cdot \beta^{-1}$ is not a square modulo roots of unity in $E$. Theorem 6 states that $\eta_{\{2\}}, \eta_{\{-1,-3\}}$ and $\eta_{\{-2,-3\}}$ form a $\mathbb{Z}$-basis of $\tilde{C}$. We have

$$
\begin{aligned}
\eta_{\{2\}} & =(1+\sqrt{2})^{-1}=\zeta^{3} \cdot \gamma, \\
\eta_{\{-1,-3\}} & =1-\zeta^{2}=\zeta \cdot \alpha \cdot \beta^{-1} \cdot \gamma, \\
\eta_{\{-2,-3\}} & =\alpha \cdot \beta .
\end{aligned}
$$

The determinant of the transition matrix gives the index $[C: \tilde{C}]=2$ for $k$, which equals the upper bound given by Proposition 5 .

This research was supported by the Grant Agency of the Czech Republic by the project $201 / 07 / 0191$.

\section{References}

[1] R. Kučera, On bases of the Stickelberger ideal and of the group of circular units of a cyclotomic field, J. Number Theory 40 (1992), 284-316.

[2] - On the Stickelberger ideal and circular units of a compositum of quadratic fields, ibid. 56 (1996), 139-166.

[3] - A note on Sinnott's definition of circular units of an abelian field, ibid. 63 (1997), 403-407.

[4] Z. Polický, On the index of circular units in the full group of units of a compositum of quadratic fields, ibid. 128 (2008), 1074-1090.

[5] W. Sinnott, On the Stickelberger ideal and circular units of a cyclotomic field, Ann. of Math. 108 (1978), 107-134.

Department of Mathematics and Statistics

Faculty of Science

Masaryk University

Kotlářská 2

61137 Brno, Czech Republic

E-mail: alize@seznam.cz 